\title{
Alpha production in humans under conditions of false feedback
}

\author{
ERNEST LINDHOLM and STEVEN LOWRY \\ Arizona State University, Tempe, Arizona 85281
}

\begin{abstract}
Subjects were pretrained in either Jacobson's relaxation or a control relaxation technique, then served in four daily sessions of biofeedback training. On some days, the feedback was veridical with respect to alpha production, while on other days, the feedback falsely indicated either success or failure at the control task. The results showed that alpha increased over trials, but this increase was independent of feedback contingency. Subjective reports of mood were not influenced by feedback falsely indicating success or failure at the control task, and there were no reliable relationships between mood and amount of alpha actually produced. Prior training on Jacobson's relaxation did not enhance alpha production. It is concluded that alpha production and positive mood states are not systematically related and that neither of these variables is operantly conditioned through biofeedback.
\end{abstract}

Brown (1970, 1971, 1974), Kamiya (1968, 1969) and others have reported that humans can learn to control their alpha production and that successful learning is accompanied by a variety of positive mood states (e.g., euphoria, well-being) collectively referred to as the "alpha experience." Others (e.g., Lynch, Paskewitz, \& Orne, 1974) have argued that alpha production is not learned, that rather, subjects habituate to the strangeness of the experimental environment and over time, engage in fewer activities that tend to block alpha, such as visual fixation on objects in the room. Regarding mood states, Plotkin, Mazer, and Lowey (1976) found no relationship between mood and the amount of alpha produced, although they suggested the possibility that the subjects' perceived success or failure might influence subjective reports of mood; that is, subjects who thought they were successful might report more positive mood states than subjects who thought they were not successful at the control task.

The Lynch et al. (1974) position that alpha enhancement is not learned is questioned by the results of Brolund and Schallow (1976), who showed that combining feedback and reward led to greater alpha enhancement than feedback alone, suggesting that experimental contingencies were controlling the behavior (alpha production) to some reliable degree. Further, Lynch et al. (1974) employed a noncontingent feedback control in which the noncontingent feedback of one subject is a replay of the contingent feedback received by another. This may well be inappropriate for alpha learning experiments, since subjects do (by whatever means) increase alpha over trials, thus the replay of increasing density of feedback over trials might serve to fortuitously reinforce almost anything the control subject does which works in the direction of increasing alpha. This could introduce a bias in the direction of reducing group differences between contingent and noncontingent groups and obscure real differences between groups.

The other type of control group used in alpha experiments is the no-feedback control, which simply requires subjects to sit in a comfortable chair in a quiet room for the duration of the experiment (Browland \& Schallow, 1976). The problem here is that these subjects receive less stimulation than subjects receiving feedback (contingent or otherwise) and thus might display enhanced alpha due to a movement toward a sleep or semisleep state. It appears, therefore, that while some argue that alpha enhancement is not a learned phenomenon, the lack of appropriate control groups detracts from the force of these arguments.

There appear to be four important questions that require evaluation before the worth of alpha conditioning experiments can be adequately evaluated: (1) Are alpha increases learned as a result of experimental contingencies? (2) Is the "alpha experience" closely related to the amount of alpha actually produced? (3) Is the intensity of the "alpha experience" influenced by the subject's perceived success or failure? (4) Is the amount of alpha produced and the intensity of the "alpha experience" influenced by the subject's ability to relax? There is an implicit assumption that alpha density and relaxation are closely related, yet this assumption has never been directly tested by manipulating relaxation as an independent variable.

\section{METHOD}

\begin{abstract}
Subjects
Ten male and 10 female subjects were solicited by advertisement to participate in an experiment in which they could learn self-relaxation techniques and control of brainwaves. All were members of psychology classes regularly offered at Arizona State University.
\end{abstract}




\section{Apparatus}

Brain activity was recorded from occipital-occipital placements referenced to left mastoid using Grass silver disk electrodes and Grass electrode paste. Signals were amplified by a Beckman Type 411 dynograph, the high-level output of which was fed to the input of a DEC-LAB-8 computer that was programmed to detect alpha activity according to the following criteria: (1) There must be $1 \frac{1 / 2}{2}$ cycles of activity falling within the $8-$ to $12-\mathrm{Hz}$ frequency band, and (2) the amplitude of the activity must exceed the Schmitt trigger threshold set for each subject during the baseline sessions. When both criteria were met, the computer software turned on the biofeedback display which consisted of a $61 \times 61 \mathrm{~cm}$ sheet of milk-white Plexiglas located $61 \mathrm{~cm}$ from the subject's face and back-lighted by a 15-W ac bulb. The Plexiglas formed the face of a plywood box thus providing a diffuse low-intensity glow that covered a large portion of the visual field and minimized focusing.

\section{Procedure}

Relaxation training. The 20 subjects were divided into two groups of 5 males and 5 females each. The subjects in the Jacobson's group received $3 \mathrm{~h}$ of relaxation training, as did the subjects in the control relaxation group; the latter were told only to listen to white noise played at a low intensity and to relax as best they could.

Biofeedback training. Four 54-min sessions of biofeedback training were employed. Subjects were told that each session investigated a different brainwave, and that recent research indicated that relaxation enhanced control of all brainwaves, thus they should use their prior relaxation training to keep the feedback light on as much as possible. This was deception. In fact, the four sessions operated as follows: Contingent Day 1 (CD1) was the first day of feedback for all subjects. Feedback was contingent on alpha production, and this was true also for the fourth day (Contingent Day 4, abbreviated CD4). Days 2 and 3 were either false increasing (FI) or false decreasing (FD) feedback, counterbalanced across subjects. Under FI, the feedback density started at a low level and increased over trials, while the reverse was true for the FD condition. To make the illusion of "real" feedback convincing, the amounts delivered on Days 2 and 3 were based on individual subject's Day 1 performance, and were programmed for delivery according to an unpredictable computerized schedule, the only constraint being that feedback increased or decreased over trials for the FI and FD groups, respectively. Thus, the amount of feedback presented to each individual subject was both a familiar and credible amount, referenced to his or her Day 1 performance. The rationale for this particular design was as follows: The first day must be contingent to establish baseline performance, and the last day should also be contingent to assess timedependent changes in alpha production. The middle 2 days were FI and FD to assess perceived success on mood and also to determine whether feedback contingency was systematically related to alpha production.

\section{RESULTS AND DISCUSSION}

The .95 confidence level was adopted throughout as indicating the presence of a reliable difference.

The percent alpha produced during all phases of the experiment are displayed in Figure 1. All functions superficially resemble "learning curves," since performance during Trial 4 was generally superior to performance during Trial 1. An ANOVA revealed main effects for trials and rest vs. feedback. The main effect

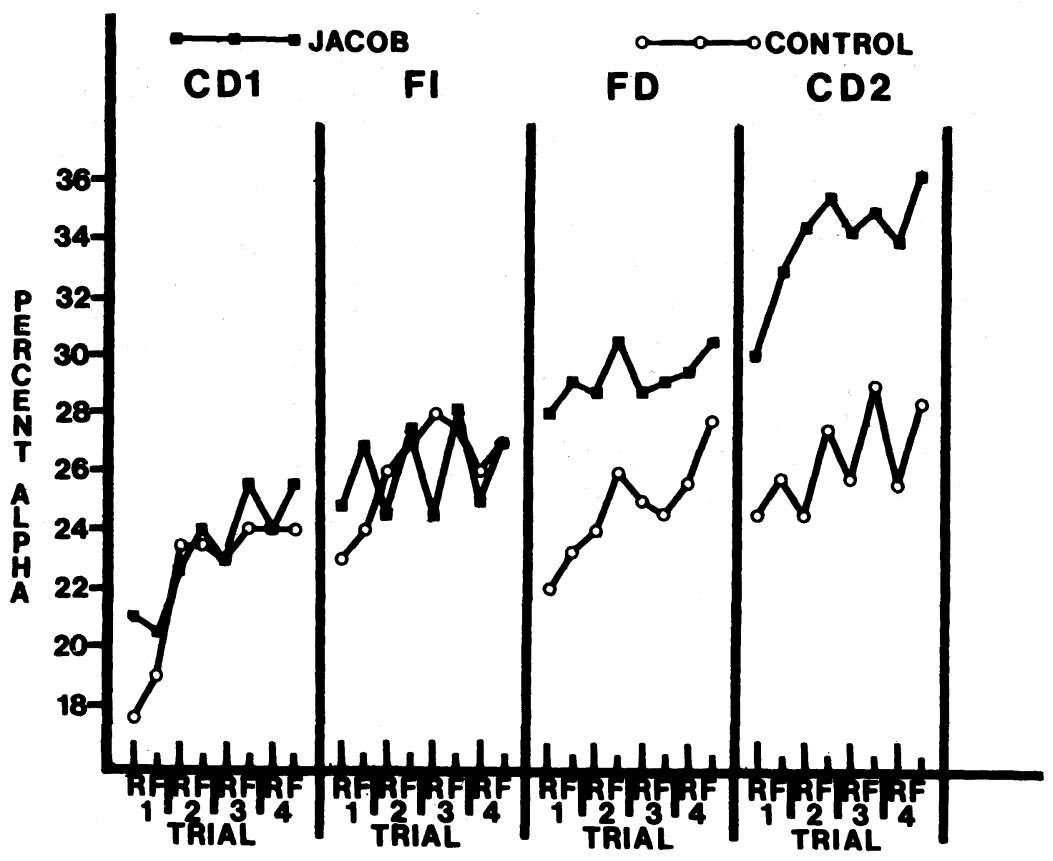

Figure 1. Percent alpha produced by the Jacobson's relaxation group (solid squares) and the control relaxation group (open circles) as a function of sessions and trials. Abbreviations: CD1 and CD2, Contingent Days 1 and 2, respectively; FI and FD, false increasing and false decreasing biofeedback, respectively. $R$ and $F$, rest and feedback, respectively. The first $R$ period in each session is the mean of the baseline recording period. 
for sessions approached the .05 level and was therefore further analyzed by comparing CD1 alone with CD2, as a test for extreme differences. This analysis also failed to detect reliable sessions differences. No other main effect or interaction approached significance in either ANOVA.

The interpretation of these results is straightforward: The Jacobson's relaxation and control relaxation groups behaved similarly throughout the experiment. Both displayed more alpha as a function of trials and both displayed more alpha during feedback than during rest periods. However, the amount of alpha produced was not affected by whether the feedback was veridical or false, demonstrating that alpha production was not under the control of the experimental contingencies. We also analyzed the data from the viewpoint of net alpha change, for example, alpha produced during each feedback period minus alpha produced during the preceding rest period. This "change score" produced functions (not shown) that varied in a nonsystematic manner and remained close to 0\% change. An ANOVA in this case produced no main effects or interactions that approached reliability.

The mood scores (responses on the Mood Adjective Check List, MACL, Nowlis, 1965) were analyzed by ANOVA, Mann-Whitney U, and parametric and nonparametric correlational methods. None of these analyses even hinted at a reliable relationship among the variables of amount of alpha actually produced, subjective report of mood, prior exposure to relaxation training, or perceived success or failure on the control task. Differences attributable to sex of subject were sought in all of the above analyses, but none were found.

We purposely used visual feedback since the early positive reports of alpha conditioning used visual feedback while more recent, negative findings employed nonvisual feedback designs. Also, we designed control groups that we feel eliminated or circumvented the problems inherent in the control groups used by others. Additionally, we used a computerized system for detecting alpha activity that circumvented the "roll off" problems inherent in the "active filter" systems used by others. Finally, we gathered data on our subjects over a 4-day period (plus relaxation pretraining) which is longer than other published experiments. Nonetheless, there was no evidence that changes in alpha production were learned through operant conditioning, since the response (alpha production) was not under the control of reinforcement contingencies (biofeedback being veridical or false). We did replicate Brown's (1971) finding that alpha production is greater during feedback than during rest periods. While Brown interpreted this finding as evidence for alpha learning, we point out that feedback need not be contingent to produce this effect, and if alpha is considered to be change scores, no evidence for learning is apparent. Finally, the "alpha experience" appears to be nothing more than suggestibility; empirically, mood was not related to the amount of alpha produced nor to perceived success or failure.

\section{REFERENCES}

Brolund, J. W., \& Schallow, J. R. The effects of reward on occipital alpha facilitation by biofeedback. Psychophysiology, 1976, 13, 236-241.

Brown, B. B. Recognition of aspects of consciousness through association with EEG activity represented by a light signal. Psychophysiology, 1970, 6, 442-452.

Brown, B. B. Awareness of EEG subjective activity relationships within a closed feedback system. Psychophysiology, 1971, 7, 451-464.

Brown, B. B. New mind, new body. New York: Harper \& Row, 1974.

KamiYa, J. Conscious control of brainwaves. Psychology Today, 1968, 1, 57-60.

KamiYA, J. Operant control of the EEG alpha rhythm and some of its reported effects on consciousness. In C. Tart (Ed.), Altered states of consciousness: $A$ book of readings. New York: Wiley, 1969.

Lynch, J. J., Paskewitz, D. A., \& Orne, M. T. Some factors in the feedback control of human alpha rhythm. Psychosomatic Medicine, 1974, 36, 399-410.

NowLIs, V. Research with the mood adjective check list. In S. S. Tomkins \& C. E. Izard (Eds.), Affect, cognition, and personality. New York: Springer, 1965.

Plotkin, W. B., Mazer, C., \& Lowey, D. Alpha enhancement and the likelihood of an alpha experience. Psychophysiology, 1976, 13, 466-471.

(Received for publication November 9, 1977.) 